\title{
Ion-plus Salinity Gradient Flow Battery
}

\author{
Chenxiao Jiang ${ }^{1}$, Ying $\mathrm{Mei}^{2}$, Xianhui $\mathrm{Li}^{1}$, Zhe Yang${ }^{1}$, Hao Guo ${ }^{1}$, Senlin Shao ${ }^{1}$, Siew-Chong \\ $\mathrm{Tan}^{3}$, Tongwen $\mathrm{Xu}^{4}$, and Chuyang Y. Tang ${ }^{1}$ \\ ${ }^{1}$ University of Hong Kong Department of Civil Engineering \\ ${ }^{2}$ University of Hong Kong \\ ${ }^{3}$ University of Hong Kong Department of Electrical and Electronic Engineering \\ ${ }^{4}$ University of Sci. and Tech. of China
}

July 22, 2020

\begin{abstract}
A Salinity Gradient Flow Battery (SGFB) is a novel type of battery that utilizes the selective ion-transition across ion exchange membranes to produce Donnan potential and stores energy in the form of salinity gradient power (SGP). Nevertheless, the commercialization of SGFB had been halted because of the low charging-discharging efficiency due to the relatively high ohmic solution resistance in the low salinity solution. By considering the contribution of supporting ions in a redox flow battery (RFB), we proposed an "Ion-plus SGFB" system by internally-integrating the RFB and SGFB system for the breakthrough of SGFB recycling efficiency. The supporting ions in the salinity solutions overcome the trade-off limitation between Donnan potential and internal-resistance without sacrificing on the SGP, and increase the power density to $1.15 \mathrm{~W}$ cm- 2 which is two times higher than control system $(0.45 \mathrm{~W} \mathrm{~cm}-2)$. The novel designed system is environmental-friendly and gentle for ion exchange membrane
\end{abstract}

\section{Hosted file}

Manuscript_Plain text_aiche J.docx available at https://authorea.com/users/345342/articles/ 471596-ion-plus-salinity-gradient-flow-battery 